Int Arch Allergy Immunol 1996;111:35

\title{
Human Eosinophil Granule
}

\begin{tabular}{l|l|l} 
A.M. & Ann M. & Dvorak
\end{tabular}

Department of Pathology, Beth Israel Hospital, Harvard Medical School, Boston, Mass., USA

Correspondence to: Ann M. Dvorak, M. D., Department of Pathology, Beth Israel Hospital, Harvard Medical School, 330 Brookline Avenue, Boston, MA 02215 (USA)

Electron micrograph $(\times 94,000)$ of an intracellular human eosinophil granule in an ileal biopsy of a patient with ulcerative colitis. This granule is a representative of the major granule population in eosinophils and is composed of a central, electron-dense crystalline area and a less dense matrix surrounding the core. A trilaminar membrane encloses the granule. Of particular interest is the presence of an elongated, narrow extension of this granule that primarily contains electrondense core material (known to contain major basic protein). The unit membrane overlying this granule extension comes in contact with the plasma membrane of this eosinophil (closed arrow). A plasma membrane-connected coated vesicle is nearby (open arrow). Numerous larger, smooth membrane-bound vesicles reside in the cytoplasm surrounding this granule.

Interstitial collagen fibers are aligned below the plasma membrane of this eosinophil. 


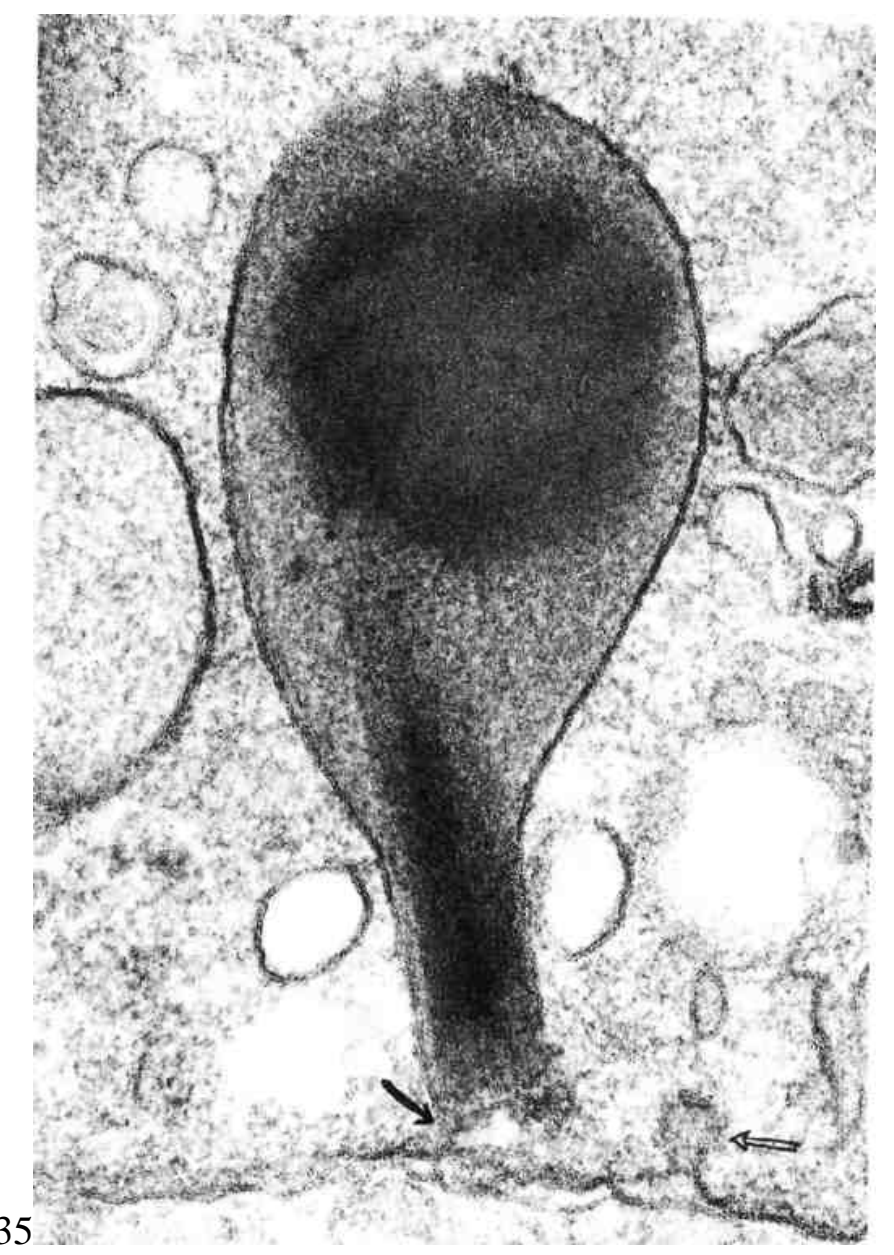

